\title{
Optical education of mathematical students at M. V. Lomonosov Moscow Sate University
}

\section{Valerii Kandidov, Nikolai Koroteev, Vladimir Makarov}

Valerii P. Kandidov, Nikolai I. Koroteev, Vladimir A. Makarov, "Optical education of mathematical students at M. V. Lomonosov Moscow Sate University," Proc. SPIE 2525, 1995 International Conference on Education in Optics, (13 October 1995); doi: 10.1117/12.224072

Event: SPIE's 1995 International Symposium on Optical Science, Engineering, and Instrumentation, 1995, San Diego, CA, United States 


\title{
Optical education of mathematical students at M. V. Lomonosov Moscow State University
}

\author{
V. P. Kandidov, N. I. Koroteev, V. A. Makarov \\ Physics Department \& International Laser Center, M. V. Lomonosov Moscow State University \\ Vorobievy Gory, Moscow 119899, RUSSIA
}

\begin{abstract}
The concept of optical education of students in the Departments of Mathematics and Mechanics and in the Department of Computer Science and Cybernetics of Moscow State University is discussed. Methods of teaching of different aspects of optics in these departments are largely determined by a high mathematical training of students.
\end{abstract}

Keywords: teaching scheme, mathematical models of optical phenomena, inductive method of presentation, computer-assisted demonstrations

\section{INTRODUCTION}

M.V. Lomonosov Moscow State University (MSU) was founded in 1755 and since that time has been playing an outstanding role in education, science and culture in Russia. At the present moment there are more than 8,000 professors, lectures and research associates working at MSU. There are 125 members of Russian Academy of Sciences (including several Nobel Prize winners) and over 6,000 Doctors of Science and Candidates of Science ( $\mathrm{Ph}$. D.). More than 26,000 undergraduate students and about 5,000 graduate students study in 19 departments (or faculties) of MSU. Each department consists of a number of chairs (branches) headed by prominent professors and scientists. Every branch conducts scientific research and some of them are known not only for teaching but also for fruitful research activity. MSU has become the first Russian university to have the special status of autonomy and self-governing.

The oldest university of Russia has a long-time tradition of mathematical education. Since its foundation the university has been having courses in mathematics. The Department of Physics and Mathematics was set up in 1804. Its development has led to the creation of Department of Mathematics and Mechanics (DMM) in 1933, Department of Physics (DP) in 1933, and Department of Computer Science and Cybernetics (DCSC) in 1970. The school of mathematics in Moscow State University is world known for the works of Nicolai Lusin, Andrei Kolmogorov, Ivan Petrovskii, Andrei Tikhonov, Mstislav Keldysh and other prominent scientists.

Nowadays, DMM is one of the main centers of mathematical thought. It consists of two parts: Division of mathematics (15 branches) and Division of mechanics ( 8 branches). More than 2,000 undergraduate students study during 10 semesters for Master Degree. The department staff consists of nearly 350 professors, lectures and well-qualified researchers. Beginning with the third year of study student's research is carried out supervised by a professor of the Department in a special field of science.

DCSC separated from MDD 25 years ago. It trains more than 1,700 students for Bachelor Degree (8 semesters) and, after that, for Master Degree (4 semesters). DCSC consists of 14 branches and 14 scientific laboratories. An integral part of the work of the Department workers is fundamental and applied research in 
fields of Computer Systems, Mathematical Methods of Physics, Mathematical Statistics, and Informatics. Beginning with the third year of study, students major in special fields of science and receive deeper training in them. At the present time there are 7 members of Russian Academy of Sciences and over 300 professors, Doctors of Science and Candidates of Science (Ph. D.) working at the Department of Computer Science and Cybernetics.

It is necessary to stress a very high educational level of students studying in these mathematical Departments. According to statistic data, about one fourth of undergraduate students are recommended by Academic Council to proceed with studying and continue their education further at the postgraduate level. Annually, the students publish about 100 articles in various scientific journals.

\section{TEACHING SCHEME}

Physics courses at the mathematical Departments of MSU have the aim of giving to the students the general education in Sciences on one hand, and providing a more specialized education in Physics on the other hand. These courses present fundamental laws, basic ideas and methods of classic and modern Physics. They also combine studying of natural physical phenomena and development of intuition in Physics with high level of education which is necessary for future mathematicians.

The courses of Physics at these mathematical Departments, DMM and DCSC, are taught one and a half and two years, respectively. In DMM the course of Physics structurally includes the following sections: Electrodynamics (72 hours), Quantum Mechanics (64 hours), and Statistical Physics (72 hours). Four academic hours every week include one lecture ( 2 hours) and 2 hours of practical studies (a seminar). It takes approximately $3 \%$ of the total time allotted for all education process at this Department. Theoretical Optics is included into the first two parts. Besides these sections, the students attend the courses which present the basis of modern Mechanics.

In DCSC the whole course of General and Theoretical Physics (it takes 5\% of time in the teaching scheme) is conventionally divided into several sections: Mechanics (72 hours), Electricity and Magnetism (64 hours), Statistical Physics and Thermodynamics (72 hours), Quantum Mechanics ( 32 hours), and Physics of Wave Processes (72 hours). The first and the second sections are delivered to all students. Only the students who specialize in the fields of Systematic Analysis, Mathematical Statistics, and System Programming have course of Statistical Physics and Thermodynamics. The students study Quantum Mechanics and Physics of Wave Processes if they are interested in Mathematical Physics, Computer Methods, Research Automatization, Nonlinear Dynamic Systems and Processes of Control, and General Mathematics. Students of these branches are taught the different parts of Theoretical Optics included in the sections of Electricity and Magnetism [one lecture ( 2 hours) and one seminar ( 2 hours) every week], and Physics of Wave Processes ( 36 lecture hours, 2 colloquia and 36 hours of consultations for students). In accordance with our rules each student receives $2-4$ problems of different degree of difficulty. They are obligated to work through the problems themselves and present their own understanding while submitting the results to the tutors. This method helps undergraduate students to learn working under specific optics problems making use of necessary scientific literature and reference sources. We also suggest optional problems for computer simulation. On tutorial seminars teachers analyze typical problems, answer students' questions concerning their individual work. Two mid-term colloquia are meant to present solutions of the problems and discuss physics behind them. The optical education of these students takes $70 \%$ of the total time allotted for the whole Physics course. 


\section{METHODS OF TEACHING}

Methods of teaching the different aspects of optics are mainly based on both the student's good knowledge of Mathematics obtained at the University and their knowledge of Physics gathered in school. Formation of the integral notion about fundamental principles of light interaction with matter and getting familiarity with the basic ideas of modern optics are based on the use of methods of Mathematical Physics. Special emphasis is placed upon construction of mathematical models of optical phenomena, and physical interpretation of the results obtained. Great attention is paid to the problem of the results being adequate to the real processes. One of the goals of this course is to show students close connection between physical ideas and corresponding mathematical models used to describe them. During the lecture course the inductive method of presentation which is characteristic for courses of General Physics and the deductive method generally used in courses of Theoretical Physics are efficiently combined. Such approach is of special importance for optical education of students specializing in Mathematics.

\section{PROGRAM OF OPTICAL EDUCATION}

At the both Departments we start from Maxwell system of equations for electromagnetic field in a medium and the material equations for optical spectral region and introduce the wave equation for electric field strength, the expressions for energy and Poynting vector. Solution of wave equation is discussed in details. Using the boundary conditions for temporal and spatial Fourier components of electromagnetic field we consider the problem of light reflection from the surface of an isotropic medium (Snellius law, Fresnel formulae). After that, the notions of spatial and time coherence are introduced. Using simple examples we discuss such problems as interference, Huygens-Fresnel principle, diffraction and dispersion of light. We obtain a parabolic equation for slowly varying amplitude of the wave and consider propagation of light with Gaussian profile at the boundary of the medium. In the average, it takes 3-4 lectures (in the 7-th semester) to deliver to high-qualified in Mathematics students of DMM the parts of Theoretical Optics mentioned above. We cannot completely realize this program in 4 semesters at DCSC because its students at the moment of presentation have not finished the study of Methods of Mathematical Physics and Differential Equations. We are just working on these problems at the beginning of the course 'Physics of Wave Processes'.

Here we represent an example of the lecture course program named 'Physics of Wave Processes' given last year at DCSC.

Lecture 1. TRAVELLING WAVES. Definition of wave and wave process, transmission of information and energy by the wave. Problem on wave propagation. One-dimensional wave equation; factorization of the wave equation. Simple harmonic wave, the spatial and temporal scales. Plane, cylindrical and spherical waves. Longitudinal and transverse waves. Physical explanation of Courant condition for stability of finitedifference schemes for the wave equation.

Lecture 2. SOUND WAVES. A set of hydrodynamics equations, the boundary conditions. Linear acoustics approach, wave equation, speed of sound, velocity potential. Sound waves on the boundary of two media; matching conditions, wave resistance of the medium. A flux of acoustic energy and volume energy. Intensity of sound, measuring the relative loudness of sounds, decibel scale, numerical evaluations. Basic mathematical models for nonlinear waves in acoustics, simple waves equations, Korteweg-de Vries equation, Burgers equation; laws of conservation. 
Lecture 3. ELECTROMAGNETIC WAVES. Formulation of the problem in case of ideal dielectric. Speed of light. Linear, circular and elliptical wave polarizations; natural light. Electromagnetic energy. Intensity. Matching conditions on the electromagnetic waves on the boundary of two media; wave resistance of the medium, relative reflection. Electromagnetic wavelength range. Estimations: solar constant, the climate on the Earth, laser thermonuclear fusion, super-strong light fields.

Lecture 4. MODULATED WAVES. Means of transmission of information. Amplitude modulation, frequency spectrum, broadcasting. Superposition of equidistant harmonics. Amplitude of quasi-harmonic signal. Pulse duration and its spectral bandwidth. Mode-locked laser.

SPECTRAL ANALYSIS. Spectrum of periodical signal, Gibbs' oscillations. A pulse spectrum. Integral Fourier transformation.

Lecture 5. DISCRETE FOURIER TRANSFORM. Do harmonics really exist? Power-density spectrum. Damped vibrations spectrum. Fourier transform of discretely sampled data. Properties of discrete Fourier transform, the division signal and its spectrum into periods. Nyquist critical frequency for the computation grid. Frequency aliasing.

Lecture 6. DISPERSION. First-order approximation of the dispersion theory, group velocity. Transfer equation of wave packet, running time. Normal and anomalous dispersion, Rayleigh formula. Second-order approximation of the dispersion theory. Scalar wave equation in parabolic approximation. Pulse spreading. Bit-rate.

Lecture 7. SPATIAL DISPERSION. Waves in chains, the transparency band, Nyquist frequency. Dispersion of difference schemes for wave equation. Wave guide.

DISPERSION IN TIME-DOMAIN. Electronic theory of dispersion of light, line absorption, anomalous dispersion. Wave dispersion in plasma.

Lecture 8. REFRACTION AND REFLECTION OF WAVES. Formulation of the problem in case of wave incident on the boundary of two media at an angle. Boundary conditions. Refraction and reflection laws; phenomena of total internal reflection in Nature and manmade devices. Fiber optics, fiber communication lines.

Lecture 9. WAVE INTERFERENCE. Interference of two monochromatic waves, superposition of plane waves, the interference fringe width, interference of waves emitted by point sources. Fringes with thin films, anti-reflection coating, multi-layer filters. Michelson interferometer. Standing waves.

Lecture 10. COHERENCE. Origin of fringes. Fringes with quasi-monochromatic light. Wave coherence and visibility of fringes, the complex degree of coherence. Coherence time and length. Wiener-Khintchine theorem. Fourier spectroscopy.

Lecture 11. MULTIPLE-BEAM INTERFERENCE. Interference of waves from aligned sources of radiation. Array antenna, directionality control. Spectral instruments. Fabry-Perot etalon.

Lecture 12. DIFFRACTION. Mathematical statement of the diffraction problem. Helmholtz-Kirchhoff integral theorem. Sommerfeld principle of limiting amplitude. Kirchhoff approximation. Fresnel-Kirchhoff diffraction formula. Secondary disturbance. Huygens-Fresnel principle. Diffraction pattern of a circular aperture. Fresnel's zones. 
Lecture 13. PARABOLIC APPROXIMATION IN DIFFRACTION THEORY. Fresnel diffraction. Wave equation in the parabolic approximation, the derivation of this equation. Diffraction of plane waves, an angular spectrum. Diffraction by a slit. Analogy between beam diffraction and dispersive spreading of the pulse, frequency and angular spectra. Near-field and far-field zone of diffraction, the geometrical optics approximation. Beam divergence. Gaussian beam. Diffraction grating.

Lecture 14. FOURIER-OPTICS. Lens as a Fourier processor. Spatial filtering. Elementary theory of holography.

THE EMISSION OF LIGHT. Classic model of an atom. Bohr atom, spontaneous and stimulated radiation.

Lecture 15. LASER RADIATION. Coherent amplification, the generation threshold. Operation of an laser. Laser levels diagram. Laser radiation characteristics. Types of lasers, their features. Laser applications in sciences and technology.

Lecture 16. BASIC MATHEMATICAL MODELS IN NONLINEAR WAVE OPTICS. Wave interaction in nonlinear dispersive media, phase matching. Formulation of the problem in case of harmonics generation, parametric interaction in the plane wave approximation. Self-action of light waves. Nonlinear Schrödinger equation, pulse self-phase modulation and beam self-focusing. Laws of conservation and their role in the numerical simulations. An overview of basic methods for numerical investigations.

It can be seen that in the course 'Physics of Wave Processes' the optical teaching is carried out in close connection with general analysis of wave phenomena in Nature. The course includes main notions of wave theory such as plane wave, harmonic wave, polarization, energy flow, wave impedance. The physical picture of the wave propagation takes the central position in our course. We use so-called "electro-mechanical analogy" of wave processes as one of the important components of forming the notion about general laws in nature. The conception of this course can be considered as further development of well-known Berkeley's course of Physics. Much attention is given to spectral analysis because this method is widely used in optics and in numerical experiments. The equivalency of spectral and temporal approaches in wave processes presentation is shown. The fundamental law connecting spectral width and wave duration which is wellknown as "Theorem of frequency width" is emphasized in all the course. Use of spectral method allows to consider wave propagation and traditional numerical schemes from similar points of view. A bright example of it can be seen in analysis of spatial dispersion of the wave in discrete structures (chains) and dispersion of the finite-difference numerical schemes for wave equation.

Along with classical optics we discuss some problems of nonlinear optics. For solving the problem of light propagation in nonlinear medium two approaches are considered: a spectral and a field ones. Basic mathematical models concerning generation of optical harmonics, phenomena of pulse self-phase modulation, beam self-focusing are presented within the framework of the spectral approach based on wave synchronism in a medium with dispersion. Some problems of fiber optics are also included.

In parallel with wave propagation in a nonlinear medium we discuss a few methods of numerical integration of the Nonlinear Schrödinger equation. On the basis of the field approach it is possible to develop computer simulation of modern problems of ultra-short light pulses. Some parts of Theoretical Optics at the both Departments are delivered in the course of Quantum Mechanics (quantum theory of light generation, quantum theory of dispersion etc.). They take about $15 \%$ of total time allotted for this course. 


\section{COMPUTER-ASSISTED DEMONSTRATIONS}

The course presentation is accompanied by physical demonstrations of wave processes in optics, acoustics, mechanics, and radio physics. Lecture demonstrations are useful for illustration of wave propagation in elastic medium, transmission of information by light wave, phenomena of electromagnetic wave polarization, formation of wave packet by harmonics addition. They are used to demonstrate normal and anomalous dispersion, phenomena of interference and diffraction, total internal reflection of electromagnetic waves, wave propagation in optical wave guide and fiber, and high sensitivity of interferometric measurements. Some experimental demonstrations on laser physics show light generation in gas laser, high power density and small divergence of laser radiation, and nonlinear effects of second harmonic generation and stimulated Raman scattering.

We efficiently combine physical demonstrations with computer demonstrations of physical phenomena. We believe that for Computer Science students it is of great importance to be familiar with computer abilities in simulations of physical phenomena. A lecturer takes the opportunity of putting together theoretical presentations, physical demonstrations, and numerical experiment. The library of programs for PC contains at the present time about 20 computer demonstrations on general physics.

Below we present several short descriptions of computer demonstrations on wave optics from the library of programs of the course "Physics of Wave Processes".

Traveling and standing waves: The synchronous dynamic picture of plane waves transmission is presented. It shows time-dependent behavior of waves, running to the left and to the right, standing wave which is the sum of the previous ones.

EM-field strength in electromagnetic wave: The first part of the demonstration illustrates mutually orthogonality of electric and magnetic strength vectors, and also of wave vector in a plane electromagnetic wave, the dynamics of spatial and temporal variation of EM-field strength vectors. The second part of the demonstration is the dynamic illustration of temporal variation of the electric field strength vector for different wave polarization of light (linear, circular, elliptical). This vector is given in the projection on the plane, perpendicular to the direction of transmission.

Monochromatic light waves on the boundary of two media: The demonstration gives a comprehensive impression of harmonic wave propagation through the boundary of two media. It is possible to observe incident, reflected, and refracted waves at the different moments of time.

\section{CONCLUSIONS}

Since 1984 the Chair of General Physics and Wave Processes of Physucs Department of MSU has been realizing these forms of optical education of mathematical students at M. V. Lomonosov Moscow State University. We are proud of our modest contribution to preparing high-class grduates successfully working in the field of computer simulation in nonlinear optics.

The authors would like to note the great contribution of Profs. A. V. Andreev, V. I. Shmalhausen and Drs. A. A. Karabutov, T. A. Matveeva, and S. A. Shlenov to optical education of mathematical students. 